\title{
Evidencia clínica de los nuevos dispositivos en estudios randomizados y registros: ¿cuánto tiempo necesitamos para definirla y cuánto para incorporarlos a la práctica cotidiana?
}

\section{Clinical evidence with new devices from randomized studies and registries: When would be the ideal period of time to introduce them in clinical practice?}

En 2002, al presentar los resultados al año del estudio RAVEL, ${ }^{1}$ muchos de nosotros nos preguntábamos, al observar los datos angiográficos del estudio, si esto significaba el fin de la reestenosis coronaria y probablemente también el fin de la cirugía de revascularización miocárdica (CABG). Los números presentados por ese estudio por entonces parecían contundentes y llamaban a ser entusiastas: $0 \%$ de reestenosis y casi $97 \%$ de ausencia de eventos clínicos adversos al año, incluyendo muerte, infarto y nuevas revascularizaciones, todos eran números que animaban a soñar. El estudio SIRIUS, con el mismo diseño de DES, reportó un año después similares contundentes resultados, ${ }^{2}$ que fueron corroborados por los estudios realizados con el stent liberador de paclitaxel. ${ }^{3-4}$ Muy pocos sin embargo tomaron en cuenta el escaso sustento biológico en experimentación animal previa que tuvieron los dos primeros diseños de stents farmacológicos y los potenciales riesgos que podrían tener en la evolución clínica alejada la incidencia de stent trombosis, fenómeno de muy baja prevalencia, aunque de incidencia constante aun años después del implante..$^{5-7}$

La historia que vino después es conocida por todos.

Hoy, a más de 10 años de aquellas experiencias, advertimos que los resultados de estudios aleatorizados y/o registros ${ }^{8-9}$ con estos mismos devices comparados con la CABG nos muestran realidades completamente diferentes: las diferencias en la tasa de revascularización con respecto a la CABG siguen siendo significativamente elevadas, estas diferencias significativas ahora se extienden a la incidencia en muerte e infarto de miocardio (IM) existiendo además un marcado incremento de eventos cardíacos adversos mayores luego del primer año de seguimiento. ${ }^{8-11}$

No viene al caso adjudicar responsabilidades, si acaso hubiese alguna, pero es bueno analizar cuáles pudieron ser nuestros errores en la toma de decisiones para tratar de no repetirlos. Primero debemos analizar si los resultados del SYNTAX ${ }^{8}$ y/o FREEDOM ${ }^{10}$ pudieron ser anticipados al momento de iniciar estos estudios, y si hubo algún cambio durante el seguimiento alejado que podía predecir aquellos resultados.

Si observamos los resultados de todos los estudios pivotales realizados con estos dos DES en un período alejado de seguimiento, claramente podemos concluir que los mismos hallazgos que los mencionados estudios presentan hoy ${ }^{8-10}$ estaban ya presentes en el seguimiento tardío de la mayoría de estos estudios pivotales con estos dos stents farmacológicos. En el estudio RAVEL a 5 años, si bien la incidencia de eventos adversos continuaba siendo favorables al grupo DES, las diferencias entre ambos habían disminuido $(\mathrm{p}=0,03$ a los 5 años $v$ s. $\mathrm{p}<0,0001$ en el primer año, en todos los casos a favor del grupo DES) y, muy importante, los pacientes tratados con DES a 5 años presentaron una no significativa mayor incidencia de muerte e infarto que el grupo de convencional. ${ }^{12}$ Analizando los estudios SIRIUS, se puede advertir el 
mismo efecto: incremento de eventos adversos en el seguimiento alejado; es más, el análisis conjunto de todos los estudios SIRIUS a 5 años de seguimiento ${ }^{13}$ mostró mayor mortalidad e incidencia de infarto $\mathrm{Q}$ en los pacientes diabéticos en el grupo DES comparado con el grupo tratado con BMS ( $\mathrm{p}=0,03$ para ambos a favor del BMS). Vale recordar que este diseño de stent fue uno de los seleccionados para el estudio FREEDOM. ${ }^{10}$

Similares hallazgos se obtuvieron a 5 años cuando se comparó este último diseño de stent farmacológico con terapéutica intravascular radiante en pacientes con reestenosis de un stent convencional (BMS); al año de seguimiento, hubo significativas ventajas en el stent liberador de fármacos, que se perdieron parcialmente a los 3 y completamente a los 5 años con inclusive una incidencia significativamente mayor de IM en el grupo DES. . $^{14-16}$

Si observamos los estudios con stents liberadores de paclitaxel, se puede advertir similar incremento de eventos adversos luego del primer año de seguimiento. En el análisis conjunto a 5 años de seguimiento de los estudios TAXUS I, II, IV y V, ${ }^{17}$ si bien se observó un beneficio en el número de nuevas revascularizaciones en el grupo tratado con DES, luego del primer año hubo un significativo incremento de IM $(3,8 \%$ vs. $2,3 \%$ para DES y BMS, respectivamente; $\mathrm{p}=0,03)$ y del punto compuesto de muerte cardíaca $\mathrm{e}$ $\operatorname{IM}(6,7 \%$ vs. $4,5 \%$ para DES y BMS, respectivamente; $\mathrm{p}=0,01)$. Todos estos hallazgos nos sugieren que es muy probable que los eventos adversos hoy vistos en los estudios SYNTAX y FREEDOM ${ }^{8,10}$ podrían haber sido en su mayoría predecibles al momento de iniciarse estos trials en los años 2005/2007.

Esta divergencia clínica observada con el seguimiento longitudinal de los pacientes estuvo presente también cuando vemos comparaciones entre los diferentes diseños de DES. El estudio SYRTAX, que comparo el stent Taxus con el stent Cypher, mostraba al primer año una significativa ventaja en el punto final compuesto para los pacientes tratados con Cypher; ${ }^{18}$ sin embargo, estas diferencias habían desaparecido completamente en el seguimiento a 5 años con inclusive idéntica incidencia de stent trombosis con ambos diseños de DES. ${ }^{19}$ Recientes comparaciones entre diferentes generaciones de DES siguen mostrando cuán dinámicos son los cambios en los resultados con el seguimiento alejado de estos pacientes. Por ejemplo, en el estudio LEADERS, que incluyó 1707 pacientes para comparar stent liberador de sirolimus y polímero durable (Cypher) y stent con polímero biodegradable y liberador de biolimus, a un año no mostró diferencias significativas entre ambos, pero en un nuevo análisis a los 4 años se observó una significativa diferencia de muerte e IM a favor del grupo de stent con biolimus y polímero biodegradable a partir del primer año de seguimiento. ${ }^{20}$

Estas discordancias en el beneficio obtenido entre el corto y mediano plazo fueron también vistas en el estudio PARTNERS, estudio aleatorizado en pacientes con estenosis crítica de válvula aórtica que evaluó la estrategia de remplazo percutáneo (TAVI) vs. quirúrgico de válvula aórtica: en el primer año se registraron diferencias en accidentes cerebrovasculares a favor de la cirugía a cielo abierto, pero a los 2 años de seguimiento los eventos se equipararon y no hubo diferencias entre el tratamiento percutáneo y la cirugía abierta de reemplazo quirúrgico en accidentes cerebrovasculares. ${ }^{21}$

Dadas las evidentes diferencias entre los resultados a corto, mediano y largo plazo que se observan con la introducción de nuevos dispositivos, los cardiólogos, angiólogos intervencionistas, cirujanos cardiovasculares y la industria asociada deberíamos reflexionar en conjunto en la necesidad de regular claramente cuándo debemos introducir la nueva tecnología en nuestra práctica clínica cotidiana a fin de proteger el crecimiento armonioso y constante de nuestra especialidad.

Es totalmente entendible la necesidad que muchas veces tiene la industria en recuperar en el menor tiempo posible parte de la gran inversión en recursos que siempre requiere todo proceso de investigación preclínica; igualmente, los cardiólogos intervencionistas, en el afán de contar con nuevas herramientas para el tratamiento de los pacientes, muchas veces tratamos de incorporar rápidamente nuevas tecnologías sin evaluar claramente el costo/efectividad que puede tener.

Para nosotros debería quedar muy claro, ante la evidencia antes mencionada, que un año de seguimiento clínico es indudable muy poco tiempo para evaluar la seguridad, la eficacia y el costo/beneficio para introducir un dispositivo y/o reemplazar el existente en la práctica cotidiana, y mucho menos -esto debemos enfatizarlo- para ser utilizado prematuramente en comparaciones aleatorizadas con otras estrategias de tratamiento tanto médicas como quirúrgicas de cuyos resultados dependerán nuevas normas y/o guías de indicaciones clínicas que puedan afectar seriamente la práctica de nuestra especialidad.

Cómo lo incorporemos y en qué indicaciones, deberán ser las sociedades científicas y las entidades regulatorias las que determinen el nivel de evidencia de cada una de ellas.

Recordemos las guías de tratamiento en la evidencia clínica: para ser incluidas, por ejemplo, en clase IIa con nivel de evidencia $B$, se necesitarán estudios aleatorizados, con resultados positivos para los puntos finales estudiados; si además se corroboran con metaanálisis recién pasaría a nivel de evidencia A. Es im- 
portante agregar que los puntos finales deberán ser clínicos y no meramente angiográficos, como fue visto en los casos antes mencionados. Creo que a esto deberíamos agregar el tiempo de seguimiento de los estudios analizados a fin de diferenciar niveles de evidencia de acuerdo al tiempo de seguimiento.

Un año puede representar el tiempo ideal de costo/eficacia para quien lo produce, pero sin duda podría ser muy poco para aquellos que potencialmente se beneficiarían con el tratamiento, principalmente cuando la enfermedad a tratar esté en los umbrales de bajo riesgo clínico que reportaban la mayoría de los trabajos anteriormente mencionados. Es decir, debemos introducir el concepto de "marcar" previamente el probable beneficio clínico neto sobre los potenciales riesgos teniendo en cuenta la evolución natural de los eventos a reducir. Un claro ejemplo: la rápida introducción del TAVI estaría completamente justificada pues, como todos sabemos, en este caso la patología a tratar presenta muy baja sobrevida en muy corto plazo de seguimiento, por lo cual toda medida para mejorarla es bienvenida. ${ }^{22,23}$

En resumen, el riesgo de perder una indicación clínica debería tener mucho mayor peso que el beneficio transitorio de introducir antes de tiempo el nuevo dispositivo.

\author{
Alfredo E. Rodríguez MD, PhD, FACC, FSCAI \\ arodriguez@centroceci.com.ar
}

Nota: Las opiniones editoriales son de exclusiva responsabilidad del autor.

\title{
BIBLIOGRAFÍA
}

1. Morice MC, Serruys PW, Sousa JE et all; RAVEL Study Group. Randomized Study with the Sirolimus-Coated Bx Velocity Balloon-Expandable Stent in the Treatment of Patients with de Novo Native Coronary Artery Lesions. A randomizedcomparison of a sirolimus-eluting stent with a standard stent forcoronary revascularization. N Engl J Med 2002;346:1773-80.

2. Moses JW, Leon MB, Popma JJ et al; SIRIUS Investigators. Sirolimus-eluting stents versus standard stents in patients with stenosis in a nativecoronary artery. N Engl J Med 2003;349:1315-23.

3. Colombo A, Drzewiecki J, Banning A, et al., for the TAXUS II StudyGroup. Randomized study to assess the effectiveness of slow-andmoderate-release polymer-based paclitaxel-eluting stents for coronaryartery lesions. Circulation 2003;108:788-94.

4. Stone GW, Ellis SG, CoxDA, et al., for the TAXUS-IVInvestigators. A polymerbased, paclitaxel-eluting stent in patients with coronaryartery disease. $N$ Engl J Med 2004;350:221-31.

5. BurkeSE, Lubbers NL, Chen Y-W, et al. Neointimal formation afterballooninduced vascular injury in Yucatan minipigs is reduced by oral rapamycin.J Cardiovasc Pharmacol 1999;33:829-35.

6. Virmani R, Guagliumi G, Farb A, et al. Localized hypersensitivity and late coronary thrombosis secondary to a sirolimus-eluting stent. Should we cautious? Circulation 2004;109:701-5

7. Rodriguez AE, Rodriguez-Granillo GA, Palacios IF. Late stent thrombosis: the Damocle's sword of drug eluting stents? Eurointervention. 2007 Feb; 2(4):512-7.

8. Kappetein AP, Feldman TE, MackMJ, etal. Comparison of coronary bypass surgery with drug-eluting stenting for the treatment of left main and/ or three-vessel disease: 3-year followup of the SYNTAX trial. Eur Heart $J$ 2011;32:2125-34.

9. Weintraub WS, Grau-Sepulveda MV, Weiss JM et al. Comparative effectiveness of revascularization strategies. N Engl J Med 2012. April 19;366(16):1467-10

10. FarkouhME,DomanskyM,Sleeper LA et al. Strategies for multivessel revascularization in patients with diabetes N Engl J of Med 2012.DOI: 10.1056/ NEJMoa1211585

11. Daemen J, Boersma E, Flather M et al.Long-term safety and efficacy of percutaneous coronary intervention with stenting and coronary artery bypass surgery for multivessel coronary artery disease: a meta-analysis with 5-year patient-level data from the ARTS, ERACI-II, MASS-II, and SOS trials. Circulation 2008;118:1146-54.

12. Morice MC, Serruys PW, Barragan P et al Long term clinical outcomes with sirolimus eluting stents J Am Coll Cardiol,2007;50: 1299-30413.

13. Caixeta A, Leon MB, Lansky AJ, et al. 5-year clinical outcomes after sirolimuseluting stent implantation: Insights from a patient-level pooled analysis of 4 randomized trials comparing sirolimus-eluting stents with bare-metal stents. J Am Coll Cardiol 2009;54:894-902.

14. Holmes Jr DR, Teirstein P, Satler L et al. Sirolimuselutingstents vs vascular brachytherapy for in-stent restenosis within bare-metalstents: the SISR randomized trial. JAMA 2006;295:1264-73.

15. Rodriguez AE, Waksman R. Sirolimus-Eluting Stents or Vascular Brachytherapy for In-Stent Restenosis After 3-Year Follow-up of the SISR (Sirolimus-Eluting Stent Versus Vascular Brachytherapy for In-Stent Restenosis) Trial: A call for caution. JACC Cardiovasc Interv. 2009; 2 (1):75-6

16. Alli OO, Teirstein PS, Satler L et al. Five-year followupof the Sirolimus-Eluting Stents vs Vascular Brachytherapy for Bare Metal In-Stent Restenosis (SISR) trial. Am Heart J 2012;163:438-45.

17. Stone G, Ellis A, Colombo A et al Long term safety and efficacy of paclitaxel eluting stents J Am Coll Cardiol Intv 2011;4:530-42

18. Windecker S, Remondino A, Eberli FR et al. Sirolimus eluting and paclitaxeleluting stents for coronary revascularization. NEngl JMed2005;353:653-62.

19. Räber $L$, Wohlwend $L$, Wigger $M$ et al. Five-yearclinical and angiographic outcomes of a randomized comparison of sirolimuselutingand paclitaxel-eluting stents: results of the Sirolimus-Eluting VersusPaclitaxel-Eluting Stents for Coronary Revascularization LATE trial. Circulation 2011;123:2819-28.

20. Wykrzykowska JJ, Garg S, Girasis C et al. Value ofthe SYNTAX score for risk assessment in the all-comers population of therandomized multicenter LEADERS (Limus Eluted from A Durable versus ERodableStent coating) trial. J Am Coll Cardiol 2010;56:272-7.

21. KodaliSK, Williams MR, Smith CRet al Twoyearoutcomes after transcatheter or surgical aortic-valve replacement. N Engl JMed 2012;366:1686-95.

22. Sprigings DC, Forfar JC. How should we manage sympto-matic aortic stenosis in the patient who is 80 or older? Br Heart J 1995; 74: 481-4.

23. Kini AS, Attanti S, Lee PC et al. Results of repeat balloon valvuloplasty for treatment of aortic stenosis in patients aged 59 to 104 years. Am J Cardiol. 2005; 95: 43-47. 